\title{
EDITORIAL
}

\section{Death following coronary angioplasty}

\section{H H Gray, K G Callum}

The National Confidential Enquiry into Peri-Operative Deaths (NCEPOD) recently published a study attempting to review all deaths within 30 days of a coronary angioplasty procedure in the UK between September 1998 and August 1999. What observations were made?
See end of article for authors' affiliations

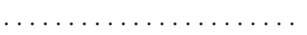

Correspondence to: Dr Huon Gray, Wessex Regional Cardiac Unit, Southampton General Hospital, Tremona Road, Southampton SO16 6YD, UK
S ince 1987 the National Confidential Enquiry into Peri-Operative Deaths (NCEPOD) has undertaken detailed peer review of the clinical details of patients who died following anaesthesia and various surgical procedures. Their annual reports have reviewed the quality of care delivered, not specifically the causes of death, and attempted to identify potentially remediable factors in clinical practice. Last year NCEPOD published a study of a percutaneous "minimally invasive" procedure attempting to review all deaths within 30 days of a coronary angioplasty (PTCA) procedure in the UK between September 1998 and August 1999. ${ }^{1}$ What observations were made?

All UK intervention centres were invited to participate, and $84 \%(n=36)$ of National Health Service (NHS)-but only $38 \%(\mathrm{n}=5)$-of private, intervention centres returned data. A total number of 21222 PTCA procedures were performed with an overall mortality calculated to be $0.87 \%$, validating the figure of $0.91 \%$ independently reported by the British Cardiovascular Intervention Society. ${ }^{2}$ All deaths occurring in hospital were identified, but data collection systems were less robust for any death occurring in the community and a few may have been missed. Data were complete for 121 deaths and these formed the main basis of the report. The majority of those who died were high risk cases before the angioplasty procedure was undertaken; a high proportion had some form of acute coronary syndrome (85\%), 62\% had PTCA undertaken for acute myocardial infarction, 63\% had moderate or severe left ventricular dysfunction, and 33\% were in cardiogenic shock. Only 10\% of the patients who died were routine elective admissions, $78 \%$ were already in-patients at the time when the decision was made to undertake PTCA, and $81 \%$ had their procedure performed as an emergency. A total of 55 (45\%) patients were transferred from another hospital for their coronary intervention, but no patient was thought to have deteriorated significantly during the actual transfer journey, tending to confirm that interhospital transfer for primary PTCA in high risk patients can be safe and feasible within a reasonable time period. ${ }^{3}$ However, while ambulance services in the UK have protocols for response times after emergency calls from outside hospital, there are currently no legal obligations for rapid transfer from one hospital to another, a situation that merits further attention.

Eighty two per cent of those who died had some coexisting medical condition, the most common of which were hypertension (38\%), diabetes $(20 \%)$, chronic obstructive airways disease (17\%), previous cerebrovascular event (14\%), and a creatinine concentration $>200 \mu \mathrm{mol} / \mathrm{l}$ $(9 \%)$. Seventy seven per cent had two or three vessel coronary artery disease, although $81 \%$ of procedures were single vessel angioplasty, most commonly involving the left anterior descending $(55 \%)$ and right $(32 \%)$ coronary arteries. When death occurred it did so early; within 24 hours of the procedure in $50 \%$ and within 3 days in $83 \%$.

\section{NATIONAL GUIDELINES FOR PTCA}

National guidelines for the practice of PTCA have been published jointly by the British Cardiac Society and the British Cardiovascular Intervention Society, ${ }^{4}$ and on those issues relating to practice which the NCEPOD survey addressed both individuals and institutions appeared to be adhering closely to them. Consultant involvement was high, the decision to carry out PTCA being made by a consultant cardiologist in $97 \%$ of cases and $79 \%$ of procedures being performed by a consultant cardiologist. The remaining $21 \%$ were undertaken by junior staff who had completed their interventional training. In only $5 \%$ of cases had the operator performed less than 60 PTCA procedures in the previous year, the minimum annual number recommended to maintain competence at the time. A consultant interventionist was immediately available in the hospital in $98 \%$ of cases. A designated and fully equipped recovery area was available in $81 \%$ of cases, and after leaving the catheter laboratory the majority of patients were cared for in some form of higher dependency area (cardiac care unit 56\%, intensive care unit 18\%, high dependency unit 6\%), the majority of the remainder going to a cardiac ward.

Regular resuscitation training for catheter laboratory staff was reported as occurring in 94\% of the questionnaires received. Centres reported that they held regular audit meetings in $92 \%$ of questionnaires, although disappointingly 23\% of the deaths reported in this study were not actually considered at such a meeting. All centres had access to emergency cardiac surgery; in 93\%

Abbreviations: $C A B G$, coronary artery bypass graft; NCEPOD, National Confidential Enquiry into Peri-Operative Deaths; NHS, National Health Service, PTCA, percutaneous transluminal coronary angioplasty 
of cases this was on the same site. Interestingly, only eight $(6.6 \%)$ of the 121 patients who died had been referred for coronary artery bypass graft (CABG) surgery before their death. Of these, only four (3.3\%) were transferred as an emergency from the catheter laboratory to the operating theatre, two underwent surgery later but within 24 hours, and a further two had surgery after more than 24 hours but during the same admission. Since this study was a review only of those who died it was not known how many patients had been referred for CABG after their angioplasty procedure and subsequently survived.

\section{"The NCEPOD study was observational and did not attempt to be rigorously scientific, being concerned mainly with factors influencing the quality of care delivered"}

The NCEPOD study was observational and did not attempt to be rigorously scientific. In common with previous NCEPOD reports it involved only those patients who died following their procedure, the proposal being that analysis of the associated clinical and institutional factors may yield useful data that might help improve overall practice. Useful observations may certainly be possible from this cohort of patients, but conclusions and extrapolation to other patient groups need to be considered carefully. With those provisos it was, nevertheless, an attempt to undertake a national survey of all deaths following coronary angioplasty procedures and resulted in $84 \%$ of NHS interventional centres contributing. The report provided an independent corroboration of the overall mortality rate reported annually by the British Cardiovascular Intervention Society. Adherence to national guidelines was also noted to be good although the report concluded that intra-aortic balloon pumps were used too infrequently $(60 \%)$ when cardiogenic shock was encountered, and both electronic and paper data collecting systems in hospitals were often inadequate. The overwhelming majority of those who died could be identified before their procedure as being at higher than average risk (severity of myocardial ischaemia, extent of coronary artery disease, and severity of ventricular dysfunction) and very few deaths followed elective procedures.

These observations support the suggestion that it may be possible prospectively to identify groups of patients at low procedural risk. Also, emergency coronary bypass surgery was undertaken in a remarkably small number of those patients who died following their PTCA procedure (3.3\%). This highlights an interesting paradox which is relevant to the proposed devolution of more angioplasty to district general hospitals without surgical facilities, a practice that has been more common in some other European countries than in the UK those who die following PTCA are likely to be higher risk patients who might not be considered ideal for new centres in the early stages of their development, and yet it is these patients who may have the least need for surgical standby. Conversely, more elective cases who are perceived to be at low procedural risk may require emergency CABG infrequently (currently around $0.5 \%$ of procedures annually in the $\mathrm{UK}^{2}$ ), but they may have the most to gain in the few cases where surgery is indicated. Transfer of patients from noninterventional to interventional centres for early angioplasty appears, at least in this high risk cohort, to be possible without jeopardy to the patient, and this may be relevant in the continuing debate about how to plan angioplasty services nationally in the UK. Individual operators and institutions currently have a high level of adherence to national guidelines for good practice. The challenge now is to refine and redefine these guidelines so that the best possible balance can be achieved between high quality care and the considerable expansion in coronary angioplasty services that will be required to achieve the aspirations laid down in the government's National Service Framework for coronary heart disease. ${ }^{7}$ By analysing the practice of interventional cardiology in the UK, at least as it applies to those who have had the worst clinical outcome following an angioplasty procedure, the NCEPOD study has made a useful contribution to this continuing debate.

\section{Authors' affiliations}

H H Gray, NCEPOD Specialty Adviser, Wessex Regional Cardiac Unit, Southampton General Hospital, Southampton, SO166YD,UK

K G Callum, NCEPOP Clinical Coordinator, NCEPOD, 35-43 Lincoln's Inn Fields, London WC2A 3PN, UK

\section{REFERENCES}

1 NCEPOD. Percutaneous transluminal coronary angioplasty: a report of the National Confidential Enquiry into Peri-Operative Deaths. 35-43 Lincoln's Inn Fields, London WC2A 3PN. www.ncepod.org.uk

2 de Belder MA. Cardiac intervention procedures in the United Kingdom 1997: developments in data collection. Council of the British Cardiovascular Intervention Society (www.bcis.org.uk). Heart 2000;82 (suppl 2):II2-9.

3 Straumann E, Yoon S, Naegeli B, et al. Hospital transfer for primary coronary angioplasty in high risk patients with acute myocardial infarction. Heart 1999;82:415-9.

4 Parker DJ, Gray HH, Balcon R, et al, on behalf of the British Cardiac Society (www.bcs.com) and British Cardiovascular Intervention Society (www.bcis.org) Working Group on Interventional Cardiology. Planning for coronary angioplasty: guidelines for training and continuing competence. Heart 1996;75:419-25.

5 Gray HH, Swanton RH, Schofield PM, et al. Coronary angioplasty: guidelines for good practice and training. Joint working group on coronary angioplasty of the British Cardiac Society (www.bcs.com) and British Cardiovascular Intervention Society. Heart 2000;83:224-35.

6 Michalis LK, Stroumbis CS, Pappas K, et al. Treatment of refractory unstable angina in geographically isolated areas without cardiac surgery. Invasive versus conservative strategy (TRUCS study). Eur Heart J 2000;21:1954-9.

7 Anon. The National Service Framework for coronary heart disease. London: Department of Health, 2000. www.doh.gov.uk/nsf/ coronary.htm

\section{COMMENTARY}

This editorial summarises the results of the interesting confidential enquiry (NCEPOD) into death resulting from PTCA. It has to be viewed with some caution. Firstly, the data collection was not complete and refers only to those deaths reported rather than those obtained from a systematic follow up of patients out to the 30 day mark that is the conventional cut-off for perioperative mortality. Secondly, only $38 \%$ of the private centres and $84 \%$ of the NHS (state) centres in the UK contributed, and the cynic would say that the centres not reporting their results are likely to be those with the worst results. Finally, there is a surprisingly low surgery rate reported in a situation where surgery as a last resort might be expected. This may be a true finding or it may be that the deaths that occurred after a patent was taken over by the surgeons were not captured because they occurred outside the catheterisation laboratory and cardiology unit.

Despite these shortcomings the most striking finding is the very low mortality of elective rather than emergency or urgent PTCA. If these results are confirmed by a more complete survey this would be powerful ammunition for those arguing for a more widespread implementation of elective PTCA without on-site surgical cover. It is hoped that any future study of this type will address the problems of data collection since strong conclusions on incomplete data should be avoided at all costs. 